\title{
Clinical, ultrasonographic, mammographic, and histologic features of leiomyoma of the breast
}

\author{
Christopher H. Chu' ${ }^{1}$ Abdelaziz A. Atwez ${ }^{2}$ and James M. Nottingham ${ }^{2 *}$
}

${ }^{*}$ Correspondence: james.nottingham@uscmed.sc.edu

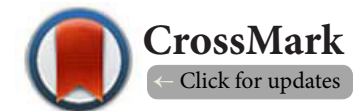

'University of South Carolina School of Medicine, Columbia, SC, USA.

${ }^{2}$ Department of Surgery, University of South Carolina School of Medicine, Columbia, SC, USA.

\begin{abstract}
Leiomyomas are benign, smooth muscle tumors classically found in the uterus, esophagus, or small bowel. In very rare instances, they can be found in the breast. We report a case of leiomyoma of the breast in a 68-year-old African American female along with the lesion's histologic, immunohistochemical, and radiologic characteristics as well as a review of the literature.
\end{abstract}

Keywords: Breast neoplasms, immunohistochemistry, leiomyoma, mammography, ultrasonography, breast cancer, breast tumor

\section{Introduction}

Leiomyomas are benign neoplasms arising from smooth muscle. These tumors are classically found in the uterus, esophagus, and small bowel. Leiomyomas are the most common mesenchymal neoplasm and also the most common benign neoplasm in the uterus [1]. Uterine fibroids, also very common, have a prevalence as high as $14.1 \%$ in women age 40-49 [2]. Likewise, in the small bowel, leiomyomas account for as much as a quarter of benign neoplasms [3].

Leiomyomas of the breast are rarely reported. These breast lesions come in two varieties: subareolar and intraparenchymal. While it is known that the origin of subareolar leiomyomas are from smooth muscle tissue from smooth muscle tissue from that area of the breast, the origin of intraparenchymal lesions remains up to debate. It is contended that they could be from teratoid origin, embryologically displaced smooth muscle from the nipple, angiomatous smooth muscle, multipotent mesenchymal cells, or myoepithelial cells [4].

Diagnosis of leiomyomas may be challenging, particularly if there is an unusual location or pattern of growth, but malignant transformation into leiomyosarcoma (LMS) is very rare with limited cases reported [5]. The prevalence of LMS accounts for only $0.0006 \%$ of breast malignancies [6]. The clinical presentation of LMS is very similar to other stromal tumors such that pre-operative diagnosis is difficult. Histopathological and immunohistochemical staining are required for the final diagnosis [7]. Stains of leiomyomas, likewise, can be compared to neighboring tissue by localizing growth factors such as transforming growth factor alpha and epidermal growth factor [8].

Breast leiomyomas are exceptionally rare, with as few as 32 cases reported in recent literature [9]. Here, we report one case of parenchymal leiomyoma of the breast with the description of the clinical, radiographic, and histologic findings.

\section{Case presentation}

The patient is a 68-year-old African American female who presented with a palpable right breast mass. She had no family history of breast, ovarian, or other cancers in the family. The mass had not grown in size but become more apparent to her. The mass was non-tender and firm to palpation. The mass moved from the chest wall, and there were no overlying skin changes.

The patient underwent a diagnostic mammogram (Figure 1A) and right breast ultrasound (Figure 1B). Both revealed a $1.0 \mathrm{~cm}$ oval mass that was partially well-circumscribed and hypoechoic. The ultrasound images were not consistent with a simple cyst nor a sebaceous cyst. These studies were her first, and there were none prior with to compare. The mass was located at the $4 \mathrm{o}$ ' clock position about $5.0 \mathrm{~cm}$ from the nipple in the substance of the breast and not attached to the chest wall. The posterior margin was slightly irregular, and one or two spiculations were seen with ultrasound. As the ultrasound was not diagnostic of a benign process and there were some worrisome features, she subsequently had a core biopsy done with three 14 gauge cores performed under ultrasound. 
A needle core biopsy with ultrasound guidance was obtained that revealed no malignant cells. Pathologic diagnosis identified nodular spindle cell proliferation with smooth muscle cell differentiation (Figure 2A). The proliferation shows low cellularity but mild cytologic atypia. Immunohistochemical staining confirmed that the mass was smooth muscle in nature with positive results for desmin (Figure 2B), SMA, calponin, and vimentin. The mass showed no mitoses, and a

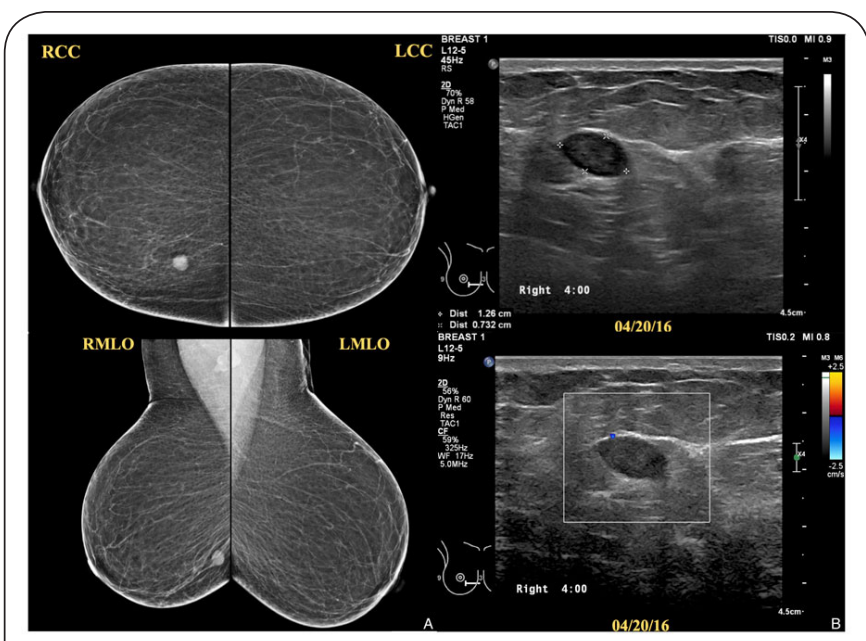

Figure 1. Radiologic findings for the breast mass.

(A) Mammograms of the right breast show a well-

circumscribed, round $1.0 \mathrm{~cm}$ mass in the lower inner quadrant.

(B) Breast ultrasonography revealed a $1.26 \mathrm{~cm} \times 0.73 \mathrm{~cm}$

hypoechoic mass with distinct margins at the 4 o' clock position

$5.0 \mathrm{~cm}$ from the nipple; color Doppler revealed no vascularity in the mass.

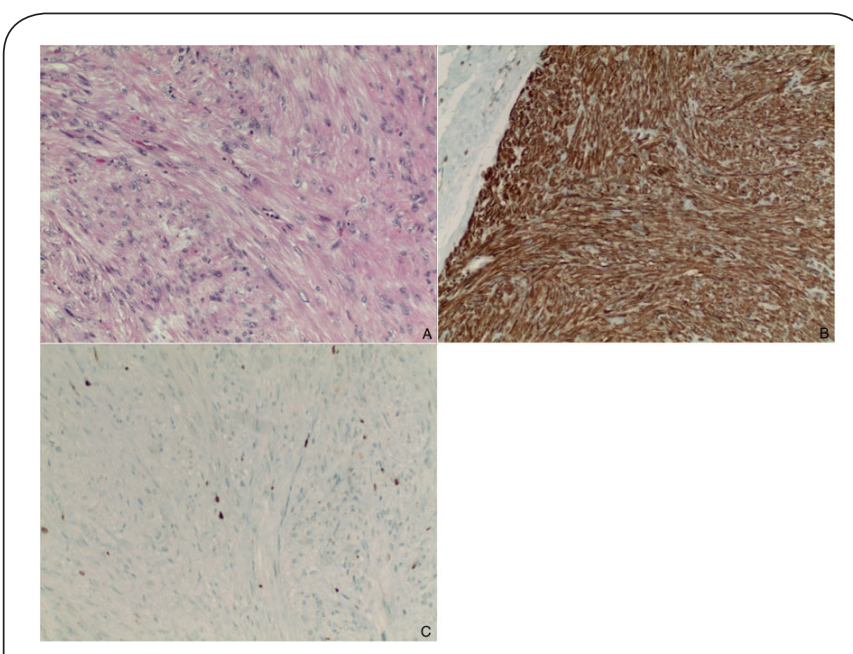

Figure 2. Microscopic findings of the right breast mass.

(A) Breast biopsy shows fibrofatty tissue composed of well-

circumscribed spindle cell forming fascicles.

(B) Immunohistochemical staining for desmin shows diffuse positive staining of the cells.

(C) Immunohistochemical staining for Ki-67 showed a low proliferative rate. low proliferative rate for $\mathrm{Ki}-67$ (Figure 2C).

Cells were negative for myogenin, CK-AE1/AE3, CK-7, CK$5 / 6$, S-100, and CD31. No evidence was found of any foci of epithelial differentiation that would otherwise suggest a metaplastic carcinoma or phyllodes tumor. The findings were found to be consistent with leiomyoma of the breast, and the pathology recommendation was that of complete excision for further examination and prevention of continued growth.

Based on clinical findings, the mammogram report, and needle core biopsy, circumferential excision was performed around a palpable lesion that extended to the breast wall. Grossly, the specimen weighed $41 \mathrm{~g}$ and contained a $1 \times 1 \times 0.8 \mathrm{~cm}$ circumscribed, pink-tan lesion. Cross-sections of the excised lesion demonstrated fragments of fibrofatty tissue composed of well-circumscribed spindle cells forming fascicles. The specimen had mild cytologic atypia without mitotic figures. No necrosis or overt features of malignancy were identified. Immunohistochemical stains performed on the excised lesion stained positively for desmin and SMA with a Ki67 of $<5 \%$ of the spindled cells. These findings led to the diagnosis of intraparenchymal leiomyoma of the breast.

\section{Discussion}

Primary leiomyoma of the breast is a very rare entity that tends to occur in the subareolar area of the breast. In contrast to leiomyosarcoma, where atypia and mitotic figures are diagnostic features, leiomyomas are characterized by collagen-forming uniform spindle cells, mature fat cells, and varying degrees of myxoid change [10].

Clinically, patients will present with a slow-growing, painless breast lump. On mammography, homogeneous, dense masses appear without calcification or cystic components [11]. The final diagnosis is confirmed with immunohistochemistry, where the smooth muscle markers consistent with leiomyoma include various forms of myosin, desmin, calponin, a-smooth muscle actin, and the heavy weight isoform of caldesmon [12]. The findings of the tumor in this case report are consistent with such diagnostic procedures and criteria.

The differential diagnosis of this tumor is complicated and includes carcinoma, sarcoma, benign tumors, and tumor-like conditions, such as an abscess. It is important to differentiate leiomyoma from its malignant counterpart, the leiomyosarcoma. Each diagnosis carries a significantly different treatment and prognosis. For a leiomyoma, the treatment is a complete excision, and recurrence is unusual [13].

\section{Conclusion}

Absolute care should be in the diagnosis of leiomyoma of the breast. Given the differential for such a presentation, the workup for a patient presenting with similar characteristics should be thorough. Palpation and imaging studies are not sufficient for diagnosis. Following a simple excision with free margins, a histology examination with the appropriate immunohistochemistry is indispensable for an accurate diagnosis. 
Chu et al. Journal of Histology \& Histopathology 2017, http://www.hoajonline.com/journals/pdf/2055-091X-4-2.pdf

\section{Competing interests}

The authors declare that they have no competing interests.

\section{Authors' contributions}

\begin{tabular}{|l|c|c|c|}
\hline Authors' contributions & CHC & AAA & JMN \\
\hline Research concept and design & $\checkmark$ & $\checkmark$ & $\checkmark$ \\
\hline Collection and/or assembly of data & -- & -- & -- \\
\hline Data analysis and interpretation & -- & -- & -- \\
\hline Writing the article & $\checkmark$ & -- & $\checkmark$ \\
\hline Critical revision of the article & $\checkmark$ & $\checkmark$ & $\checkmark$ \\
\hline Final approval of article & $\checkmark$ & $\checkmark$ & $\checkmark$ \\
\hline Statistical analysis & -- & -- & -- \\
\hline
\end{tabular}

\section{Acknowledgement}

The authors thank Dr. Michael Hayes for helpful discussion and analysis of the histopathology slides.

\section{Publication history}

Editor: Gaetano Giuseppe Magro, University of Catania, Italy. Received: 24-Jan-2017 Final Revised: 23-Feb-2017

Accepted: 10-Mar-2017 Published: 23-Mar-2017

\section{References}

1. Levy and Angela D. Esophagus: Benign Neoplasms. In Abdominal Imaging. 2013; 149-158.

2. Zimmermann A, Bernuit D, Gerlinger C, Schaefers M and Geppert $K$. Prevalence, symptoms and management of uterine fibroids: an international internet-based survey of $\mathbf{2 1 , 7 4 6}$ women. BMC Womens Health. 2012; 12:6. | Article I PubMed Abstract | PubMed FullText

3. Lee YT. Leiomyosarcoma of the gastro-intestinal tract: general pattern of metastasis and recurrence. Cancer Treat Rev. 1983; 10:91-101. | PubMed

4. Diaz-Arias AA, Hurt MA, Loy TS, Seeger RM and Bickel JT. Leiomyoma of the breast. Hum Pathol. 1989; 20:396-9. | PubMed

5. Mayerhofer K, Obermair A, Windbichler G, Petru E, Kaider A, Hefler L, Czerwenka K, Leodolter S and Kainz C. Leiomyosarcoma of the uterus: a clinicopathologic multicenter study of 71 cases. Gynecol Oncol. 1999; 74:196-201. | Article | PubMed

6. Adem C, Reynolds C, Ingle JN and Nascimento AG. Primary breast sarcoma: clinicopathologic series from the Mayo Clinic and review of the literature. Br J Cancer. 2004; 91:237-41. | Article | PubMed Abstract I PubMed FullText

7. Agrawal P, Neha $G$ and Pandey BB. Primary Leiomyosarcoma of the Breast: A Rare Case Report. Archives of Breast Cancer. 2015; 2:100-103. I Article

8. Dixon D, He H and Haseman JK. Immunohistochemical localization of growth factors and their receptors in uterine leiomyomas and matched myometrium. Environ Health Perspect. 2000; 108 Suppl 5:795-802. | PubMed

9. Granic M, Stefanovic-Radovic M, Zdravkovic D, Ivanovic N, Nikolic D, Radovanovic D and Stojiljkovic M. Intraparenchimal Leiomyoma of the Breast. Arch Iran Med. 2015; 18:608-12. I Article I PubMed

10. Rakha EA, Aleskandarany MA, Lee AH and Ellis IO. An approach to the diagnosis of spindle cell lesions of the breast. Histopathology. 2016; 68:33-44. | Article | PubMed

11. Kotsuma Y, Wakasa K, Yayoi E, Kishibuchi M and Sakamoto G. A case of leiomyoma of the breast. Breast Cancer. 2001; 8:166-9. | PubMed

12. Al-Daraji W, Husain E, Zelger BG and Zelger B. A practical and comprehensive immunohistochemical approach to the diagnosis of superficial soft tissue tumors. Int J Clin Exp Pathol. 2009; 2:119-31. I PubMed Abstract | PubMed FullText
13. Heyer $H$, Ohlinger $R$, Schimming A, Schwesinger $G$ and Grunwald $\mathrm{S}$. Parenchymal leiomyoma of the breast--clinical, sonographic, mammographic and histological features. Ultraschall Med. 2006; 27:558. | Article | PubMed

\section{Citation:}

Chu CH, Atwez AA and Nottingham JM. Clinical, ultrasonographic, mammographic, and histologic features of leiomyoma of the breast. J Histol Histopathol. 2017; 4:2. http://dx.doi.org/10.7243/2055-091X-4-2 